\title{
Agricultura de precisão: um alerta à imprecisão - estudo de caso na cafeicultura
}

\author{
Leandro da Silva Almeida ${ }^{1}$, Ednaldo Carvalho Guimarães ${ }^{1}$ \\ ${ }^{1}$ Universidade Federal de Uberlândia, Uberlândia, Minas Gerais, Brasil. E-mail: almeidalean26@gmail.com, ecg@ufu.br
}

Recebido: 10/02/2017; Aceito: 26/04/2017

\section{RESUMO}

O objetivo deste trabalho foi avaliar a discrepância existente entre mapas gerados a partir da utilização da configuração padrão fornecidos por softwares de agricultura de precisão (default) comparando com os mapas nos quais se realiza criteriosamente os procedimentos necessários para o ajuste dos mapas. Para estudo foi utilizado o programa Gamma Design Software $\left(\mathrm{GS}^{+\oplus}\right)$ versão 7.0. O experimento foi conduzido em propriedade cafeeira que possui 65 ha de cafeeiro arábica (Coffea arabica L.), cultivado em um Latossolo Vermelho Distrófico (LVd), localizada no município de Araguari-MG. Foi utilizada uma malha não sistemática de um ponto para cada hectare. Os dados a principio foram submetidos, a análise fatorial exploratória, gerando três fatores. Os fatores foram submetidos à análise espacial por meio da geoestatística. Para observar as diferenças entre os mapas foi realizada uma comparação de similaridade entre as classes dos mapas obtidos, através de tabela de validação cruzada e também realizados os testes de Cramer e Tschupov. Observou-se que a coincidência entre as classes foi longe de um, mostrando grande dissimilaridade entre os mapas. Com a realização desse trabalho ficou evidente a diferença entre o mapa gerado com o default do programa e o mapa no qual os parâmetros foram ajustados.

Palavras-chave: Geoestatística. Cafeicultura de precisão. Gestão da cafeicultura. Krigagem ordinária.

\section{Precision agriculture: an alert to imprecision - case study on coffee growing}

\section{ABSTRACT}

The objective of this work was to evaluate the discrepancy between maps generated from the use of the standard configuration provided by precision agriculture software (default) compared to the maps in which carefully performs the necessary procedures for setting the maps. For the study, the Gamma Design Sotware $\left(\mathrm{GS}^{+\oplus}\right)$ version 7.0 was used. The experiment was conducted in coffee farming which has 65 ha of Arabica coffee tree (Coffea arabica L.), cultivated in a Dystrophic Red Latosol located in the municipality of Araguari-MG. A non-systematic mesh of one point was used for each hectare. The data at first underwent exploratory factor analysis, generating three factors. The factors were submitted to spatial analysis using geostatistics. To observe the differences between the maps of similarity a comparison was made, between the classes of the maps obtained, through a cross-validation table and, also performed the tests of Cramer and Tschupov. It was observed that the coincidence between classes was far from one, showing great dissimilarity between the maps. With the completion of this work showed that there was difference between the map generated with the program's default and the map on which the parameters were adjusted.

Key words: Geostatistics. Precision coffee makers. Coffee management. Ordinary kriging. 


\section{Introdução}

É de grande relevância a atividade cafeeira no contexto socioeconômico brasileiro; difundida em mais de 2,2 milhões de hectares plantados no país, sendo que destes, em torno de $78,5 \%$ são de cafeeiro arábica e o restante tipo conilon (CONAB, 2016). O agronegócio do café, no ano de 2015, movimentou com as atividades de exportação mais de US $\$ 5.555$ milhões, sendo o café o terceiro produto agrícola nos valores de exportação, ficando atrás apenas da carne de frango e do complexosoja (CONAB, 2016).

A elaboração dos mapas de fertilidade da área de cultivo permite que sejam aplicados elementos (nutrientes) de acordo com a necessidade específica e não indistintamente como tem sido realizado convencionalmente pelos produtores (BERNADI et al., 2014; COSTA et al., 2014). Com o manejo especifico, procura-se minimizar as perdas de insumos, otimizando o sistema de produção (COSTA et al., 2014). Na agricultura de precisão, o manejo sítio-específico surge em contraposição à visão tradicional que preconiza manejo baseado nas condições médias da lavoura, o que implica na adoção de tratamentos diferenciados, segundo variações na área de cultivo, diminuindo a possibilidade de subdosagens ou superdosagens de fertilizantes o que pode causar danos ao desenvolvimento da cultura, maior perda de nutrientes além da poluição de águas subterrâneas e superficiais (MOLIM et al., 2007; HURTADO et al., 2008).

A produtividade das culturas varia espacialmente, e, determinar as causas dessas variações é o desafio que enfrenta a agricultura de precisão. As variações espaciais são estudadas por intermédio de diferentes métodos estatísticos que permitem elaborar mapas e delimitar áreas de manejo diferenciadas (BORSSOI et al., 2011; GOMES et al., 2007). Embora esses métodos estatísticos permitam inferir sobre a variabilidade do solo (vertical e horizontal), a dependência espacial entre as amostras somente pode ser modelada por meio de análises geoestatísticas (MANZIONE; ZIMBACK, 2011).

Nos últimos anos, graças aos avanços científicos e tecnológicos no campo da informática e comunicação tem-se observado um grande avanço e difusão de programas e aplicativos comerciais destinados à análise e tratamento de dados aplicados aos diversos campos do conhecimento. A gama de avanços tecnológicos e consequente redução de custos, aliada à facilidade e simplicidade de uso de plataformas destinadas ao processamento da informação têm facilitado cada vez o acesso à utilização e aplicação de tecnologias de análise espacial, o que tem permitido uma crescente popularização e disseminação da utilização dessas tecnologias para aplicação nas mais diversas áreas.
Nesse contexto, as aplicações de métodos geoestatísticos ganham destaque, uma vez que, praticamente todos os softwares recentes destinados ao tratamento da informação espacial, possuem módulos destinados a fins geoestatísticos (YAMAMOTO; LANDIM, 2013).

Todavia, a associação de procedimentos de análise de dados espaciais e técnicas estatísticas requerem conhecimentos básicos no tocante às duas áreas, no sentido de conferir aos produtos gerados qualidade nas informações apresentadas e, consequentemente, aplicabilidade prática. Rosa (2011) destaca que há um descompasso existente entre a tecnologia disponível e a efetivamente utilizada para tomada de decisões. Tal contraste pode ser justificado pelo ritmo lento com que o conhecimento teórico e procedimental necessário se dissemina entre os usuários, isto pode levar o usuário a utilizar dos softwares apenas dados que são definidos automaticamente pelo mesmo (default), sem levar em conta as particularidades dos dados, procedimentos metodológicos e ajustes necessários aos modelos, o que pode resultar em modelos equivocados ou sem validade.

$\mathrm{O}$ presente trabalho teve por objetivo avaliar a discrepância existente entre produtos gerados a partir da utilização da configuração padrão fornecidos por softwares de geoestatística (default) comparando com produtos nos quais se realizou criteriosamente os procedimentos necessários. $\mathrm{O}$ procedimento comparativo foi pautado no método da Krigagem ordinária com modelo de semivariograma ajustado automaticamente no software $\mathrm{GS}^{+®}$ e o modelo ajustado a sentimento.

\section{Material e Métodos}

O trabalho foi desenvolvido em uma propriedade cafeeira, localizada no município de Araguari-MG, região de cerrado, cujas coordenadas geográficas da sede são: $18^{\circ} 32^{\prime} 55.99^{\prime \prime} \mathrm{S}, 48^{\circ} 24^{\prime} 10.46^{\prime \prime}$ O. A altitude média da propriedade é de 940 metros, e o solo classificado como Latossolo Vermelho Distrófico (LVd). A temperatura média anual é de $21,2{ }^{\circ} \mathrm{C}$, com pluviosidade média anual de $1.566 \mathrm{~mm}$.

$\mathrm{Na}$ propriedade são cultivados 65 hectares de cafeeiro (Coffea arabica L), sendo toda a área irrigada, pelo sistema de gotejamento. As amostras de solo foram coletadas na projeção do dossel da planta, na profundidade de 0 a $20 \mathrm{~cm}$, sendo utilizada uma malha amostral não sistemática de um hectare, totalizando assim 65 amostras georeferenciadas. As análises químicas do solo foram realizadas no laboratório de análise de solos (LABAS) da Universidade Federal de Uberlândia. Os atributos avaliados são apresentados na Tabela 1. 
Tabela 1. Atributos do solo avaliados

\begin{tabular}{llll}
\hline Atributo & Abreviação* & Atributo & Abreviação* \\
\hline Matéria Orgânica & $\mathrm{MOS}$ & Teor de magnésio & $\mathrm{Mg}$ \\
Cap. de troca de cátions & $\mathrm{CTC}$ & Relação Ca/V & $\mathrm{Ca} / \mathrm{V}$ \\
Pot. Hidrogênio & $\mathrm{pH}$ & Relação $\mathrm{Mg} / \mathrm{V}$ & $\mathrm{Mg} / \mathrm{V}$ \\
Saturação de bases & $\mathrm{V}$ & Relação Ca/Mg & $\mathrm{Ca} / \mathrm{Mg}$ \\
Teor de Cálcio & $\mathrm{Ca}$ & Relação $\mathrm{K} / \mathrm{V}$ & $\mathrm{K} / \mathrm{V}$ \\
Teor de potássio & $\mathrm{K}$ & Relação Ca/Mg/K & $\mathrm{Ca} / \mathrm{Mg} / \mathrm{K}$ \\
Teor de alumínio & $\mathrm{Al}$ & Relação cálcio $/$ potássio & $\mathrm{Ca} / \mathrm{K}$ \\
CTC efetiva do solo & $\mathrm{T}$ & Relação $\mathrm{Mg} / \mathrm{K}$ & $\mathrm{Mg} / \mathrm{K}$ \\
Saturação de bases & $\mathrm{SB}$ & Relação $\mathrm{H}+\mathrm{Al} / \mathrm{T}$ & $\mathrm{H}+\mathrm{Al} / \mathrm{T}$ \\
Hidrogênio + Alumínio & $\mathrm{H}+\mathrm{Al}$ & Relação Al/V & $\mathrm{Al} / \mathrm{V}$ \\
Fósforo remanescente & $\mathrm{P}-\mathrm{res}$ & & \\
\hline
\end{tabular}

* Abreviação adotada no texto.

Para realizar a análise fatorial inicialmente foi realizada a análise exploratória dos dados obtidos a fim de verificar a existência de dados discrepantes (outliers), pois a existência desse tipo de dados pode interferir significativamente nos resultados das análises estatísticas. Foi realizado também o teste de normalidade de Kolmogorov-Smirnov com significância de $5 \%$.

Atendidas as premissas da análise fatorial realizouse os seguintes procedimentos: escolha do método de extração de fatores (método de componentes principais); escolha do método de rotação de fatores (Varimax) e, por fim, análise dos fatores gerados (FIGUEIREDO FILHO; SILVA JÚNIOR, 2010).

Com os fatores obtidos pela análise fatorial e no intuito de utilizar a geoestatística para representação espacial, realizaram-se algumas verificações primordiais: análise descritiva espacial para verificar tendências; análise de dependência espacial; escolha do melhor modelo para ajuste do semivariograma. Após estas verificações para as variáveis que possuem dependência espacial aplicou-se a krigagem ordinária para a interpolação. Já para as variáveis que não apresentaram modelo estruturado de dependência espacial foi utilizado outro método de interpolação; o método de inverso do quadrado das distâncias (RIBEIRO JÚNIOR et al, 2009).

As análises geoestatísticas foram realizadas utilizando o programa Geostatistics for the Environmental Sciences - GS ${ }^{+\circledast} 7.0$ (GAMMA DESIGN SOTWARE, 2004). No ajuste de semivariogramas foram avaliados os modelos por meio do default do programa e por meio da técnica de validação cruzada (VIEIRA et al., 1983), sendo realizada a comparação entre os dois. Nos modelos de validação cruzada foram selecionados aqueles que melhor descreveram o comportamento espacial dos fatores (NANNI et al., 2011; YAMAMOTO; LANDIM, 2013).

Para avaliar a similaridade dos mapas temáticos gerados pelo default do programa $\mathrm{GS}^{+\circledR}($ GAMMA
DESIGN SOFTWARE, 2004) e os gerados a critério do pesquisador usou-se tabela de contingência para verificar se houve diferença estatística entre as duas estimativas, utilizando para este fim, os coeficientes de Cramér e Tschuprov (SIEGEL; CASTELLAN, 1988). A tabela de contingência e os coeficientes foram calculados utilizando o programa $\mathrm{R} \quad 3.2 .1 \quad$ (R DEVELOPMENT CORE TEAM, 2015).

\section{Resultados e Discussão}

Para avaliar o uso correto do software $\mathrm{GS}^{+{ }^{\circledR}}$ foram obtidos, por meio da análise fatorial exploratória, quatro fatores que apresentaram esfericidade satisfatória pelo teste de Kaiser-Meyer-Olkin (KMO). Estes quatro fatores acumulados representam 97,05 \% da variação total dos dados, sendo que foram selecionados três testes para este trabalho. Cada um dos fatores representa com maior força uma característica de interesse no solo, sendo elas: $\mathrm{pH}$; teor de potássio; CTC e Ca/Mg. O presente trabalho não irá explorar a importância destes fatores e nem as suas características, apenas será explorada a possibilidade de imprecisões na agricultura de precisão, utilizando como estudo de caso o programa de geoestatística.

Os fatores 1 e 3 apresentaram dependência espacial e demais pressupostos necessários para o uso da Krigagem, entretanto, o Fator 2 não apresentou dependência espacial, não podendo utilizar a Krigagem como interpolador, sendo necessária a utilização do método inverso do quadrado das distâncias (IQD) que, conforme Ribeiro Júnior et al. (2009) é o interpolador da estatística mais preciso. Para confirmar o pressuposto deste trabalho, os fatores 01 e 03 foram avaliados comparando o modelo de semivariograma ajustado a sentimento com o modelo gerado pelo default do programa. No caso do Fator 2, no intuito de demonstrar que o programa gera de forma automática a Krigagem, demostrou-se que o mapa pode ser gerado mesmo quando o atributo apresenta efeito pepita puro. 
O primeiro fato comum de uso inadequado da agricultura de precisão é apresentado na Figura 1, onde o default do programa, no caso especifico do $\mathrm{GS}^{+\circledast} 7.0$ (GAMMA DESIGN SOTWARE, 2004) permite gerar os mapas de correlação espacial por krigagem mesmo que o atributo em si não apresente dependência espacial, ou seja, gera-se um mapa com o estimador inadequado, fato que leva a tomada de decisão em campo, principalmente na agricultura de precisão, incoerente com a realidade. Neste caso, fica claro o desconhecimento prévio da técnica, pois falta a análise crítica do semivariograma (YAMAMOTO; LANDIM, 2013).

A Figura 2 apresenta o semivariograma do Fator 02, que apresenta efeito pepita puro (YAMAMOTO; LANDIM, 2013). Para uma situação como essa, conforme mencionado anteriormente, o correto é aplicar outra técnica de interpolação como, por exemplo, o inverso do quadrado das distâncias, cujo mapa gerado será mais coerente com a realidade que a geoestatística, já que o atributo não apresentou dependência espacial, conforme pode ser visualizado na Figura 3.

Além disso, é comum a utilização de ajustes padrões de semivariogramas dos softwares (default's) sem realizar a análise crítica destes gerando a krigagem automaticamente.

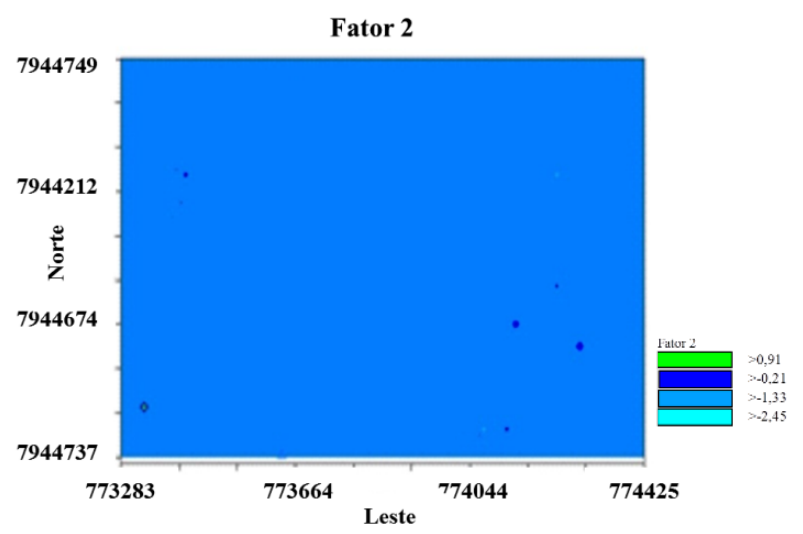

Figura 1. Mapa temático do Fator 2 gerado por Krigagem.

Fator 2

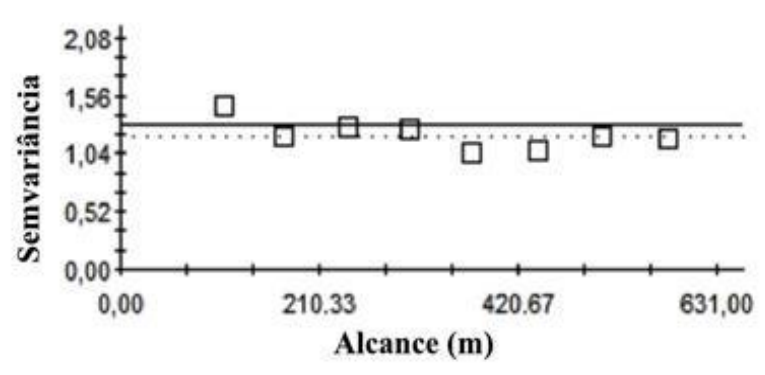

Modelo Esférico: $(\mathrm{Co}=1,3 ; \mathrm{Co}+\mathrm{C}=1,31 ; \mathrm{Ao}=\mathbf{5 8 0 , 2 3}$

Figura 2. Semivariograma ajustado para o Fator 2 (efeito pepita puro).

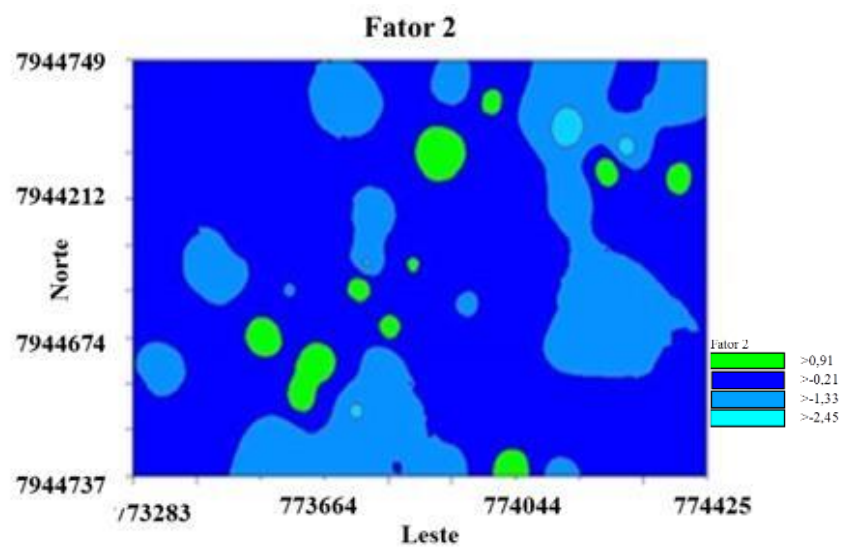

Figura 3. Mapa do fator 02 utilizando o inverso do quadrado das distâncias.

Nas Figuras de número 4 a 7 , os modelos de semivariogramas ajustados à sentimento (VIEIRA et al., 1983) e os do default do GS ${ }^{+\oplus} 7.0$ para os Fatores 1 e 3 são comparados. Observa-se que o gráfico do default do software, nos dois casos, busca sempre um efeito pepita próximo à zero, sem considerar a quantidade de pares em cada distância, algumas vezes fornecendo um peso demasiadamente importante a uma classe com um número muito reduzido de pares.

\section{Fator 1}

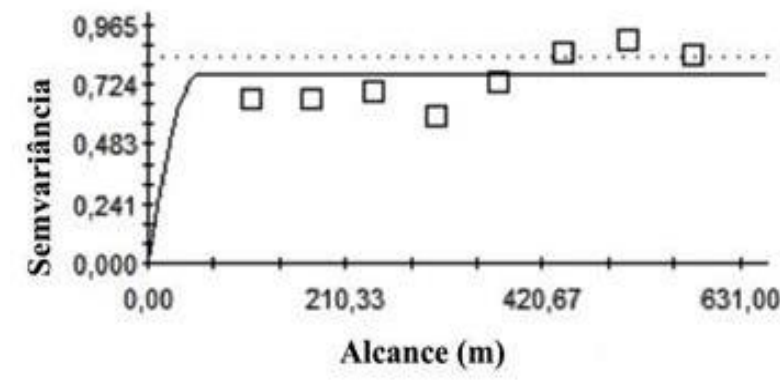

Modelo Esférico: $(\mathrm{Co}=0,001 ; \mathrm{Co}+\mathrm{C}=0,77 ; \mathrm{Ao}=\mathbf{5 3}$;

Figura 4. Semivariograma gerado pelo defaut do $\mathrm{GS}^{+\circledast}$.

Fator 1

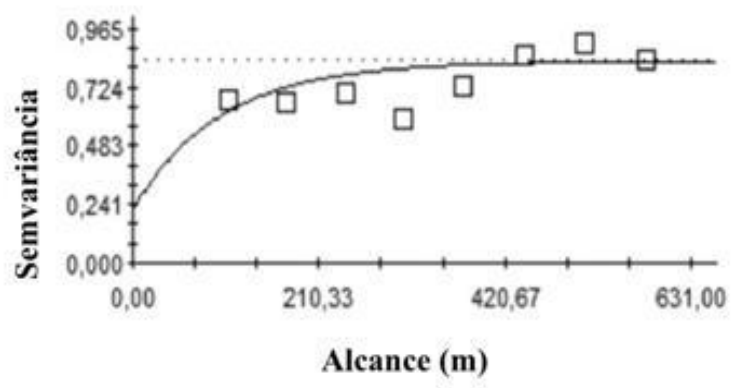

Modelo Esférico: $\left(\mathrm{Co}_{0}=0,22 ; \mathrm{Co}+\mathrm{C}=0,83 ; \mathrm{Ao}=98,33\right.$;

Figura 5. Semivariograma ajustado no $\mathrm{GS}^{+®}$. 
Fator 3

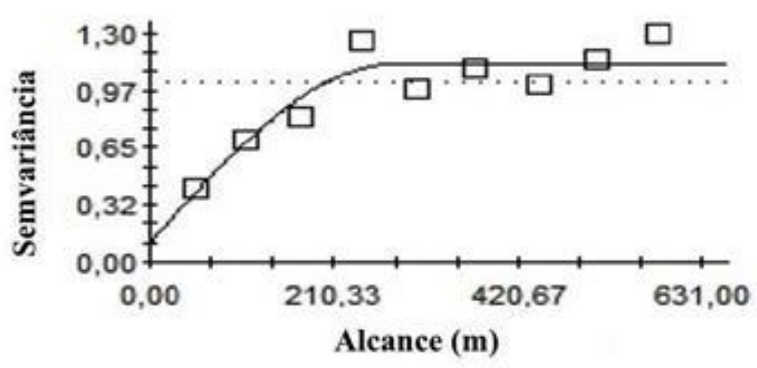

Modelo Esférico: $(\mathrm{Co}=0,11 ; \mathrm{Co}+\mathrm{C}=1,12 ; \mathrm{Ao}=277$;

Figura 6. Semivariograma gerado pelo defaut do $\mathrm{GS}^{+\circledR}$.

Fator 3

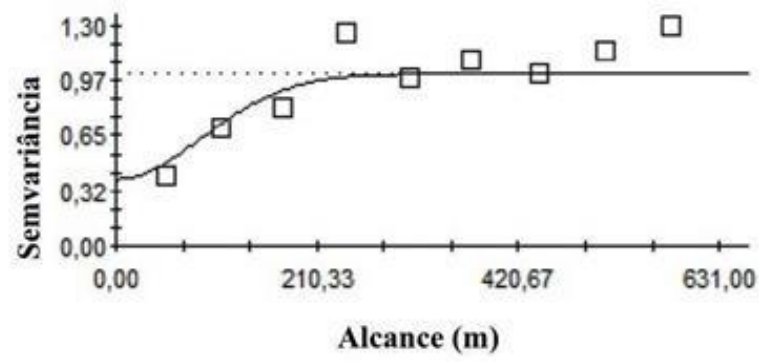

Modelo Esférico: $\left(\mathrm{Co}_{0}=0,39 ; \mathrm{Co}+\mathrm{C}=1,01 ; \mathrm{Ao}=127,37\right.$

Figura 7. Semivariograma ajustado a sentimento no $\mathrm{GS}^{+\circledast}$.

Na Tabela 2 são apresentados as classes e os pares para os fatores 01 e 03 (ambas iguais), deixando claro que não é necessário considerar a primeira classe para iniciar o ajuste do semivariograma, por conter poucos pares. Entretanto, quando o semivariograma é gerado pelo default do programa, não leva em consideração esse detalhe, forçando assim o ajuste.

Para verificar o tamanho da divergência entre ajustar o modelo a sentimento ou gerar automaticamente o semivariograma foram elaboradas as tabelas de contingência com as estimativas ( $Z$ estimados) geradas pelo default do programa, e os valores estimados ( $Z$ estimados) dos modelos ajustados a sentimento, para os Fatores 1 e 3. Para tal procedimento os valores estimados foram agrupados em quatro classes de acordo com o mapa ajustado, conforme mostrado na Tabela 3.

Os mapas gerados a partir de pressupostos díspares vão trazer divergências na distribuição espacial dos atributos na área, conforme pode ser observado nos mapas de Krigagem ordinária (Figuras 8 a 11).

Nas Tabelas 4 e 5 são apresentadas as porcentagens de coincidência para as quatro classes dos fatores $01 \mathrm{e}$ 03, respectivamente. Elaborou-se a tabela de contingência como parâmetro estatístico para verificar a diferença ou não estatística entre as duas estimativas (default e modelo ajustado), utilizando-se os coeficientes de Cramér e Tschuprov (SIEGEL; CASTELLAN, 1988) para validar a comparação. Ambos os coeficientes, cujos valores próximos de 1 indicam forte dependência (coincidência) e próximo de 0 em caso de independência (baixa coincidência).

A coincidência para nenhuma das duas classes foi alta; os coeficientes ficaram relativamente longe de 1 . A consequência desta não coincidência pode ser facilmente visualizada nos mapas de krigagem dos fatores (Figuras 8 a 11), que são muito diferentes entre si. Estas análises ressaltam a importância de se usar os softwares de maneira criteriosa, sobretudo de aplicar todo o conhecimento sobre a técnica dentro do software, pois podemos dizer que o software gera resultados com qualquer conjunto de dados que possuam as coordenadas (X e Y) e um conjunto de variáveis $(\mathrm{Z})$. Entretanto, é dever do pesquisador analisar não apenas o resultado final mas também, e principalmente, cada passo até que se chegue ao resultado final.

Este tema é muito preocupante na agricultura de precisão onde empresas adquirem os softwares e podem não ter profissional habilitado (com conhecimento necessário) para gerar os mapas de distribuição de fertilizantes e corretivos do solo com qualidade, fato que pode em certas ocasiões gerar críticas aos softwares. Frequentemente o que se pode constatar são falhas quanto ao funcionamento correto do programa e até mesmo falta de conhecimento das primícias da análise ou da maneira de aplicar o conhecimento dentro software.

A importância da aplicação de técnicas mais precisas na avaliação de atributos do solo, como a geoestatistica na atividade cafeeira vem sendo discutidas em vários estudos como os de Ferraz et al. (2012), Oliveira et al. (2008); Lima et al. (2013); Santos et al. (2015); Silva et al. (2007); Silva et al. (2010) e Silva e Lima (2012), onde os autores verificam a dependência espacial dos atributos em área cultivadas com cafeeiro destacando que as recomendações de fertilizantes e corretivos devem levar em conta a eficiência ao fazer uso da geoestatistica.

Tabela 2. Relação de classes e número de pares do semivariograma médio

\begin{tabular}{|c|c|c|c|c|c|c|c|c|c|}
\hline Classe & 1 & 2 & 3 & 4 & 5 & 6 & 7 & 8 & 9 \\
\hline Pares & 11 & 105 & 124 & 154 & 159 & 175 & 170 & 150 & 139 \\
\hline
\end{tabular}

Tabela 3. Intervalo de classes para os Fatores 01 e 03

\begin{tabular}{ccccc}
\hline \multirow{5}{*}{ Fator } & \multicolumn{5}{c}{ Classes } \\
\cline { 2 - 5 } & 1 & 2 & 3 & 4 \\
\hline 1 & $>-2.02<-1.283$ & $>-1.283<-0.545$ & $>-0.545<0.193$ & $>0.193$ \\
3 & $>-1.33<-0.49$ & $>-0.49<0.35$ & $>0.35<1.19$ & $>1.19$ \\
\hline \multicolumn{4}{l}{ Os valores representam os intervalos das classes. }
\end{tabular}




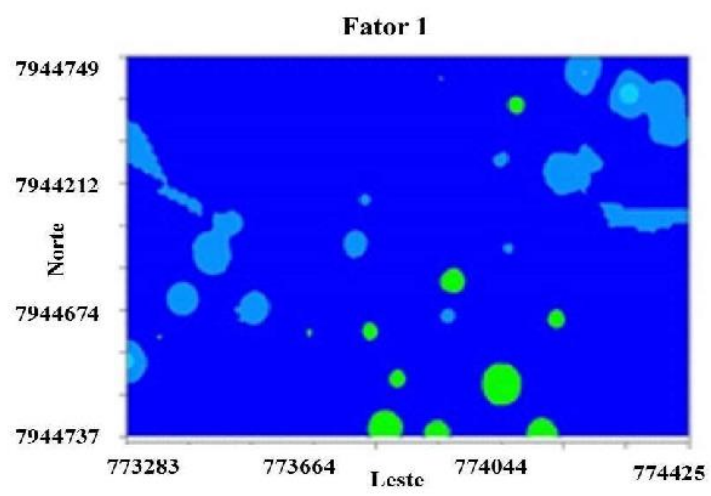

Figura 8. Krigagem sem ajuste do semivariograma

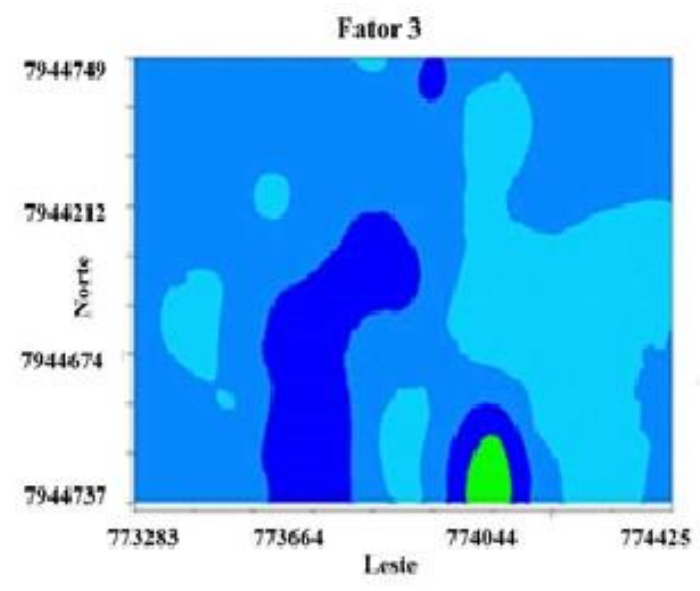

Figura 10. Krigagem sem ajuste do semivariograma

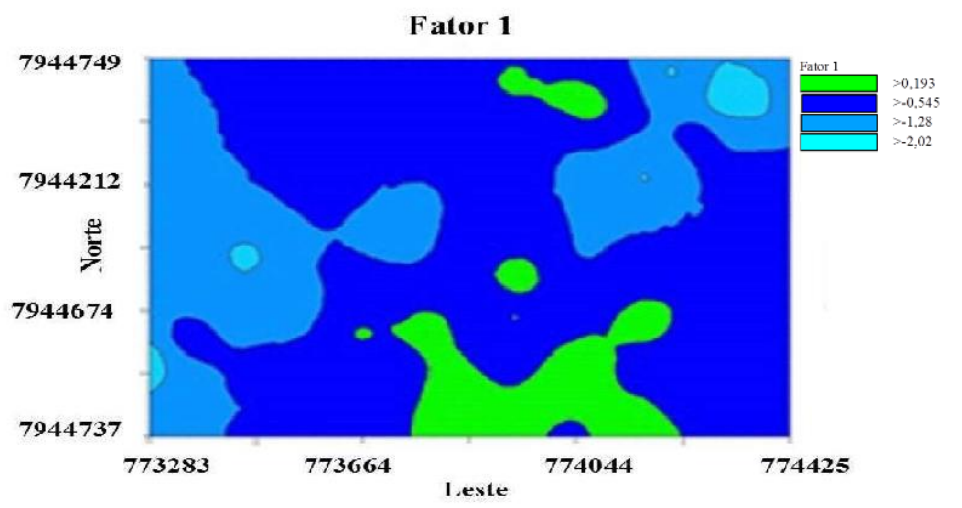

Figura 9. Krigagem com ajuste do semivariograma a sentimento

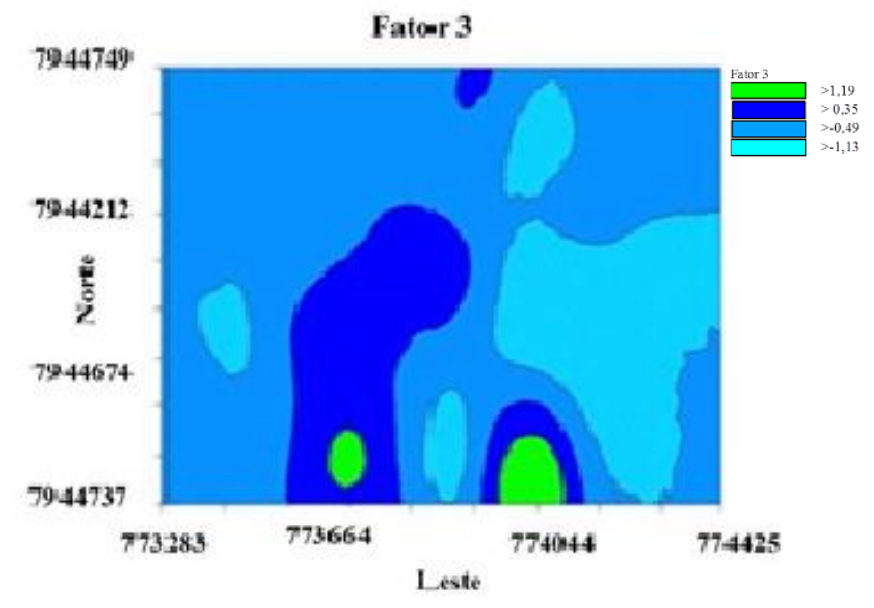

Figura 11. Krigagem com ajuste do semivariograma a sentimento
Tabela 4. Porcentagem de coincidência entre as classes nos mapas do Fator 1

\begin{tabular}{ccccc}
\hline $\begin{array}{c}\text { Tabela } \\
\text { Cruzada }\end{array}$ & 1 & 2 & 3 & 4 \\
\hline 1 & 0,43 & 1,12 & 0,00 & 0,10 \\
2 & 0,18 & 20,92 & 7,09 & 0,00 \\
3 & 0,00 & 8,83 & 50,63 & 0,54 \\
4 & 0,00 & 0,00 & 3,50 & 6,68 \\
& & \multicolumn{2}{c}{ Coeficiente Trehuprov 0,35}
\end{tabular}

Coeficiente Cramer: 0,36 Coeficiente Tschuprov 0,35

Os valores em destaque representam a coincidência entre as classes para o default do GS ${ }^{+\circledast} 7.0$ (GAMMA DESIGN SOFTWARE, 2004) e os valores dos modelos ajustados no mesmo programa.

Tabela 5. Porcentagem de coincidência entre as classes nos mapas do Fator 3.

\begin{tabular}{ccccc}
\hline $\begin{array}{c}\text { Tabela } \\
\text { Cruzada }\end{array}$ & 1 & 2 & 3 & 4 \\
\hline 1 & 19,64 & 0,07 & 0,00 & 2,06 \\
2 & 5,47 & 52,20 & 1,54 & 0,52 \\
3 & 0,00 & 0,43 & 13,96 & 1,32 \\
4 & 0,56 & 0,01 & 0,00 & 2,22 \\
\hline
\end{tabular}

Coeficiente Cramer: 0,57 Coeficiente Tschuprov 0,43

Os valores em destaque representam a coincidência entre as classes para o default do GS ${ }^{+\oplus} 7.0$ (GAMMA DESIGN SOFTWARE, 2004) e os valores dos modelos ajustados no mesmo programa.
Kravchenko e Bullock (1999), alertam que a geoestatística não é uma técnica tão fácil de ser aplicada por ter maior morosidade do que outros métodos de análise, como por exemplo, o inverso do quadrado da distância (IQD), entretanto a mesma apresenta uma descrição mais acurada dos atributos da estrutura espacial dos dados e produz a informação valiosa da distribuição da estimativa do erro requerendo, assim, maior conhecimento para o uso adequado da mesma.

Além disso, a agricultura de precisão tem trabalhado frequentemente com estatística clássica sem considerar a dependência espacial dos atributos do solo; esta variabilidade espacial vem sendo relatada desde o início do século passado por autores como Montegomery (1913) e Waynick (1918). Viera et al. (2000), em estudo sobre a geoestatistica aplicada em algumas propriedades agronômicas destacam que por volta de 1971 surgiu a geoestatística aplicada à agricultura e esta tem sido, ao longo dos anos, ferramenta importante que possibilita analisar a variabilidade do solo.

Usar os programas de geoestatística sem o devido conhecimento pode levar também a resultados que não condizem com a realidade. O default dos programas podem ajustar modelos de semivariograma, até mesmo 
se os dados não apresentaram dependência espacial. Assim, no caso da geoestatistica, é sempre importante o conhecimento técnico para avaliar o modelo de semivariograma que está sendo ajustado e que melhor se adeque para determinada situação e se realmente representa o comportamento do semivariograma.

\section{Conclusões}

Com a realização desse trabalho comparativo, fica claro que não basta apenas saber usar os softwares, é fundamental ter conhecimento dos princípios da técnica para que os resultados gerados representem a realidade da área de estudo.

No caso do uso do $\mathrm{GS}^{+\circledast}$ houve diferença considerável entre o mapa gerado com o default do programa e o mapa no qual os parâmetros foram ajustados a sentimento, deixando evidente a importância dos conhecimentos de geoestatística ao realizar esse tipo de mapeamento.

\section{Referências Bibliográficas}

BERNADI, A. C. C. NAIME, J. M.; RESENDE, A. V.; BASSOI, L. H. Agricultura de precisão: resultados de um novo olhar. Embrapa: Brasília-DF, 2014. 596 p. Disponível em: https://www.embrapa.br/instrumentacao/busca-depublicacoes/-/publicacao/1002959/agricultura-de-precisaoresultados-de-um-novo-olhar>. Acesso em 06 jul. 2016.

BORSSOI, J. A.; URIBE-OPAZO, M. A.; GALEA, M. Técnicas de diagnóstico de influência local na análise espacial da produtividade da soja. Engenharia Agrícola, JaboticabalSP, v. 31, n. 2, p. 376-387, 2011.

COSTA, N. R.; CARVALHO, M. P.; DAL BEM, E. A.; DALCHIAVOM, F. C.; CALDAS, R. R. Produtividade de laranja correlacionada com atributos químicos do solo visando a zonas específicas de manejo. Pesquisa Agropecuária Tropical, Goiânia-GO, v. 44, n. 4, p. 391-398, 2014

CONAB. COMPANHIA NACIONAL DE ABASTECIMENTO. Estimativas de Safras Agrícolas. Levantamento Café. Brasília-DF: 2016, p. 1-98. Disponível em:<http://www.conab.gov.br/>. Acesso em: 06 jan. 2016.

FERRAZ, G. A. E S.; SILVA, F. M.; CARVALHO, L. C. C.; ALVES, M. C.; FRANCO, B. C. Variabilidade espacial e temporal do fósforo, potássio e da produtividade de uma lavoura cafeeira. Engenharia Agrícola, Jaboticabal-SP, v. 32, n. 1, p. 140-150, 2012.

FIGUEIREDO FILHO, D. B.; SILVA JÚNIOR, J. A. Visão além do alcance: uma introdução à análise fatorial. Opinião Pública, Campinas-SP, v. 16, n. 1, p. 160-185, 2010

GAMMA DESIGN SOFTWARE. GS+:Geostatistics for the Environmental Sciences Versão 7.0. Gamma Design Software. Plainwell, Michigan, 2004. CD-ROM.

GOMES, N. M.; SILVA, A. M.; MELLO, C. R.; FARIA, M. A.; OLIVEIRA, P. M. Métodos de ajuste e modelos de semivariograma aplicados ao estudo da variabilidade espacial de atributos físico-hídricos do solo. Revista Brasileira de Ciência do Solo, Viçosa-MG, v. 31, n. 3, p. 435-443, 2007.

HURTADO, S. M. C.; RESENDE, A. V.; SILVA, C. A.; CORAZZA, E. J.; SHIRATSUCHI, L. S. Agricultura de Precisão: possibilidade de manejo da adubação nitrogenada para o milho no cerrado. Planaltina-SP: Embrapa Cerrados. 2008. 48 p. (Boletim, 214). Disponível em: < https://www.embrapa.br/busca-de-publicacoes//publicacao/556775/agricultura-de-precisao-possibilidades-demanejo-da-adubacao-nitrogenada-para-o-milho-no-cerrado>. Acesso em: 06 jul. 2016.

KRAVCHENKO, A.; BULLOCK, D. A comparative study of interpolation methods for mapping soil properties. Agronomy Journal, Madison, v. 91, n. 3, p. 393-400, 1999.

LIMA, J. S. S.; SILVA, S. A.; SILVA, J. M. Variabilidade espacial de atributos químicos de um Latossolo VermelhoAmarelo cultivado em plantio direto. Revista Ciência Agronômica, Fortaleza-CE, v. 44, n. 1, p. 16-23, 2013. .Disponível em: < http://www.ccarevista.ufc.br/seer/index.php/ccarevista/article/ view/1814>.

MANZIONE, R. L.; ZIMBACK, C. R. L. Análise Espacial Multivariada Aplicada na Avaliação da Fertilidade do Solo. Engenharia na Agricultura, Viçosa-MG, v. 19, n. 3, p. 227235, 2011.

MOLIN, J. P.; VIEIRA JUNIOR, P. A.; DOURADO NETO, D.; FAULIN, G. C.; MASCARIN, L. Variação espacial na produtividade de milho safrinha devido aos macronutrientes e à população de plantas. Revista Brasileira de Milho e Sorgo, Sete Lagoas-MG, v. 6, n. 3, p. 309-324, 2007. Disponível em: <http://rbms.cnpms.embrapa.br/index.php/ojs/article/view/235 >. Acesso em: 06 jul. 2016.

MONTGOMERY E. G. Experiments in wheat breeding: Experimental error in the nursery and variation in nitrogen and yield. Washington, United States: Department of Agriculture, Bureau Plant Industry, 1913. 61 p. (Bulletin n. 269)

NANNI, M. R.; POVH, S. P.; DEMATTÊ, J. A, M.; OLIVEIRA, R. B.; CHICATI, M. L.; CEZAR, E. Optimum size in grid soil sampling for variable rate application in sitespecific management. Scientia Agricola, Piracicaba-SP, v. 68, n. 3, p. 386-392, 2011.

OLIVEIRA, R. B.; LIMA, J. S. S.; XAVIER, A. C.; PASSOS, R. R.; SILVA, S. A.; SILVA, A. F. Comparação entre métodos de amostragem do solo para recomendação de calagem e adubação do cafeeiro conilon. Engenharia Agricola, Jaboticabal-SP, v. 28, n. 1, p. 176-186, 2008

R DEVELOPMENT CORE TEAM. R: A language and environment for statistical computing. Vienna: $R$ Foundation for Statistical Computing, 2011. Disponível em:< http://www.r-project.org >. Acesso em: 04 nov. 2015

ROSA, R. Análise Espacial em Geografia. Revista da ANPEGE, Presidente Prudente-SP, v. 7, n. 1, p. 275-289, 2011. Disponível em: <http://anpege.org.br/revista/ojs2.2.2/index.php/anpege08/article/view/163>. Acesso em: 07 dez. 2015. 
RIBEIRO JÚNIOR, P. J.; VIOLA, D. N.; DEMETRIO, C. G. B.; MANLY, B. F.; FERNANDES, O. A. Spatial pattern detection modeling of thrips (Thrips tabaci) on onion fields. Scientia Agricola, Piracicaba-SP, v. 66, n. 1, p. 90-99, 2009

SANTOS, E. O. J.; GONTIJO, I.; SILVA, M. B.; DRUMONT NETO, A. P. Variabilidade espacial de macronutrientes em uma lavoura de café conilon no Norte do Espírito Santo. Revista Ciência Agronômica, Fortaleza-CE, v. 46, n. 3, p. 469-476, 2015. Disponível em: < http://www.ccarevista.ufc.br/seer/index.php/ccarevista/article/ view/2204>. Acesso em: 06 jul. 2016.

SIEGEL, S.; CASTELLAN, N.J., Nonparametric statistics for the behavioral sciences, New York: McGraw-Hill, 1956, $312 \mathrm{p}$.

SILVA, F. M. SOUZA, Z. M.; FIGUEREDO, C. A. P.; MARQUES JÚNIOR, J.; MACHADO, R. V. Variabilidade espacial de atributos químicos e de produtividade na cultura do café. Ciência Rural, Santa Maria-RS, v. 37, n. 2, p. 401407, 2007.

SILVA, S. A.; LIMA, J. S. S.; XAVIER, A. C.; TEIXEIRA, M. M. Variabilidade espacial de atributos químicos de um Latossolo Vermelho-Amarelo húmico cultivado com café. Revista Brasileira de Ciência do Solo, Viçosa-MG, v. 34, n. 1, p. 15-22, 2010.
SILVA, S. A.; LIMA, J. S. S. Multivariate Analysis and Geostatistics of the Fertility of a Humic Rhodic Hapludox under Coffee Cultivation. Revista Brasileira de Ciência do Solo. Viçosa-MG, v. 36, n. 2, p. 467-474, 2012.

WAYNICK D. D. Variability in soils and its significance to past and future soil investigations. I. Statistical study of nitrification in soils. Agricultural Sciences, Davis, v. 3, n. 9, p. $243-270,1918$.

VIEIRA, S. R.; HATFIELD, J. L.; NIELSEN, D. R.; BIGGAR, J. W. Geostatistical theory and application to variability of some agronomical properties. Hilgardia, Berkeley, v. 31, n. 3, p.1-75, 1983.

VIEIRA, S. R. Geoestatística em estudos de variabilidade espacial do solo. In: NOVAIS, R. F.; ALVAREZ V., V. H.; SCHAEFFER, C. E. G. R. Tópicos em ciência do solo. Viçosa-MG: Sociedade Brasileira de Ciência do Solo, v. 1, 2000. p. $1-54$

YAMAMOTO, J. K.; LANDIM, P. M. B. Geoestatística: conceitos e aplicações. São Paulo-SP: Editora Oficina de Textos, 2013. 215 p. 